\title{
Lidil
}

Revue de linguistique et de didactique des langues

$54 \mid 2016$

La phrase en production d'écrits, approches nouvelles en didactique

\section{Claudine Moïse et Alina Opréa (dir.), Politesse et violence verbale détournée}

Revue Semen, $n^{\circ} 40,2015$

Nolwenn Bailly

(2) OpenEdition

Journals

Édition électronique

URL : http://journals.openedition.org/lidil/4132

DOI : 10.4000/lidil.4132

ISSN : $1960-6052$

Éditeur

UGA Éditions/Université Grenoble Alpes

Édition imprimée

Date de publication : 20 novembre 2016

Pagination : 209-211

ISBN : 978-2-84310-339-1

ISSN : $1146-6480$

Référence électronique

Nolwenn Bailly, «Claudine Moïse et Alina Opréa (dir.), Politesse et violence verbale détournée », Lidil [En ligne], 54 | 2016, mis en ligne le 01 janvier 2017, consulté le 31 octobre 2020. URL : http:// journals.openedition.org/lidil/4132; DOI : https://doi.org/10.4000/lidil.4132 
Réaffirmer la place de l'écriture dans les apprentissages, dans un ouvrage à la fois théorique et pratique, était un projet ambitieux. Les auteures ont tenu leur engagement d'un regard nouveau associé à l'apport d'éléments de formation, dans la lignée des années 1990 où Michel Dabène, Catherine Frier, ou Christine Barré-De Miniac ${ }^{3}$ par exemple avaient ouvert la voie de la réflexion sur l'écriture comme acculturation.

Marie-Hélène Lachaud LIDILEM, Université Grenoble Alpes

Claudine Moïse et Alina Opréa (dir.), Politesse et violence verbale détournée, revue Semen, $\mathrm{n}^{\circ}$ 40, 2015.

Si plusieurs types de violences verbales ont déjà été définies (Moïse, 2011) d'un point de vue «bio-psycho-socio-politico-linguistique» (Moïse et coll., 2015, p. 10), c'est la violence verbale détournée qui fait l'objet de ce numéro de Semen. Cette forme de violence verbale est questionnée dans son rôle au cœur des interactions. Elle s'actualise dans des interactions consensuelles et coopératives feintes et ambigües à des fins de manipulation et de harcèlement (Moïse, 2013). En outre, et c'est ainsi d'ailleurs que s'intitule ce numéro, la violence verbale détournée met à l'épreuve le système de politesse (Brown \& Levinson, 1987; Goffman, 1973; Kerbrat-Orecchioni, 1992) dans sa fonction pragmatique et perlocutoire. Les auteur-e-s se sont interrogés sur la façon dont politesse, impolitesse et violence verbale s'intriquent dans le discours. Politesse et violence verbale, bien que d'apparence antagoniste, entretiennent une relation de proximité comme en attestent de possibles formes hybrides à l'instar de l'analogie (Lucie Alexis \& Jérôme Ravat). Toute interaction peut être comprise comme une «guerre froide» (Goffman, 1973) dans laquelle la coopération des participants demeure obligatoire. Honoré de Balzac (1836) ne disait-il pas que la politesse «est une grimace sociale qui se dément aussitôt que l'intérêt trop froissé montre le bout de l'oreille»? User de violence verbale détournée lorsque l'intérêt du locuteur est froissé, lorsque sa face est menacée, est aussi et surtout un

3. Cf. Pratiques, $\mathrm{n}^{\circ}$ 105-106, 2000. 
moyen d'exercer son pouvoir face à un tiers participant qui encourage de telles pratiques.

Les médias, centre d'intérêt de ce numéro, sont donc le seul lieu mis en relief au cœur de ces articles, qui, lorsqu'ils mêlent spectacle et information, offrent aux locuteurs la possibilité de nuire à un adversaire tout en séduisant un auditoire. La violence est alors mise au service de la mise en scène (Olivier Turbide \& Marty Laforest). À la marge toutefois du numéro, la contribution de Laurence Rosier et Pierre-Nicolas Schawb questionne les relations entre les entreprises et leur clientèle, souvent «loin de l'image d'Épinal qui veut que toute entreprise doit être à l'écoute de sa clientèle et la satisfaire» (p. 119). C'est la problématique des émotions, et, dans ce cadre, le «trop-plein» émotionnel relatif à l'individu qui est avancé comme cause de la violence verbale et, de fait, de l'éviction de la politesse permettant de maintenir le lien social. Si le traitement des émotions a surtout été l'apanage de la psychologie comportementale, son étude commence à intéresser les analystes du discours en tant qu'elles font sens dans une relation d'échange. Cette dimension pathémique a également retenu l'attention de Marie Reetz dans le discours «enjoué-amical-insolent» de Cyrille Eldin, chroniqueur humoriste. Toujours est-il que seuls les médias, à fortiori avec une composante politique, ont intéressé les auteur-e-s, dans l'optique d'examiner les procédés langagiers relatifs à la violence verbale détournée à l'image de «l'attaque courtoise» (Christina Romain \& Béatrice Fracchiolla), où l'ironie et l'implicite sont des processus rhétoriques argumentatifs qui permettent au locuteur de tirer avantage de la situation. L'art de faire la cour, de séduire l'autre, en l'occurrence le tiers présent qu'est le public, est une forme de domination visant à satisfaire des intérêts particuliers. La victoire par KO verbal (Windisch, 1987) est alors possible sous forme d'une montée en tension «masquée», non fulgurante en apparence donc. L'apparence. Ce «non-totalement-dit» (Annabelle Seoane) institue au locuteur une forme de pouvoir en exerçant un contrôle sur l'autre, sans pour autant se voir affliger une «mauvaise» image. Là est la clé. La politesse garantit une «coexistence dans le dissensus » (Amossy, 2011) malgré, parfois, des ressentiments persistants (Geneviève Bernard-Barbeau).

Si la violence verbale détournée semble être particulièrement l'apanage des médias en général, surtout lorsqu'une composante politique entre en jeu, on peut se questionner toutefois sur une plus large représentation de ce type de violence. Il nous semble en effet que la violence verbale détournée prend de l'ampleur au sein de nos sociétés occidentales où il est question de prendre le pouvoir sur l'autre sans qu'il y 
ait fulgurance. Les institutions françaises en sont un bon exemple. Un numéro supplémentaire à ce sujet viendrait compléter ces analyses très enrichissantes pour la sociolinguistique française.

Nolwenn Bailly

LIDILEM, Université Grenoble Alpes

Ann-Birte Krüger, Nathalie Thamin et Stella Cambrone-Lasnes (dir.), Diversité linguistique et culturelle à l'école : accueil des élèves et formation des acteurs, revue Carnets d'atelier de sociolinguistique, $\mathrm{n}^{\circ}$ 11, L'Harmattan, 2016, 288 p.

On attendait avec impatience ce travail collectif de nos collègues de Besançon, dont une équipe, en tissage scientifique et éthique avec des équipes du LIDILEM et d'autres partenaires (CASNAV, etc.) et réseaux, travaille avec patience et méthode sur la question de la diversité culturelle et linguistique sociale, dans son rapport avec l'école.

Cet ouvrage vient à point pour répondre à notre curiosité scientifique et aux besoins du terrain enseignant - dynamiques qui se rejoignent trop rarement. Il fait suite à plusieurs actions de formation et de réflexion fécondes autour des langues dans la classe, liant avec bonheur dans une même dynamique (cf. le sous-titre) accueil des élèves et formation des enseignants - même si ces deux volets sont séparés dans l'ouvrage, ce qui n'était peut-être pas indispensable. En effet, l'introduction, fouillée et décidée en faveur d'une «école inclusive», donne résolument le ton en posant la formation des enseignants à l'accueil des enfants de la migration comme une urgence sociale : il s'agit de «dépasser l'habitus et l'idéologie monolingues» pour penser «l'altérité au sein de l'espace scolaire», d'optimiser les apprentissages communs, tout en valorisant les langues et cultures d'origine des élèves. Les auteures veulent croire à un contexte institutionnel favorable (p. 16) pour développer une formation à la diversité, du côté des élèves comme du côté des enseignants.

On apprécie particulièrement que soient détaillés des espaces et publics souvent marginalisés dans les recherches en didactique : les classes de maternelle (Cécile Goï, Rita Carol et coll., Isabelle Audras et Françoise Leclaire) ou le périscolaire (Stella Cambrone-Lannes) par exemple, les parents dans l'école (Isabelle Audras et Françoise Leclaire), les enfants sourds (Saskia Munier) par exemple. 